\title{
Qualitätskriterien in der ärztlichen Komplementärmedizin
}

Jörg Fritschi, Christoph Hänggeli

Korrespondenz: Sekretariat UNION Tribschenstrasse 7 Postfach 3065 CH-6002 Luzern Tel. 0413685805

info@unioncomed.ch

\section{Einleitung}

Bis zum 30. Juni 2005 waren die vier komplementärmedizinischen Methoden Akupunktur TCM, Homöopathie, anthroposophische Medizin und Neuraltherapie für Inhaber des entsprechenden Fähigkeitsausweises im Rahmen der Grundversicherung abrechenbar. $\mathrm{Da}$ für die Phytotherapie von der FMH kein Fähigkeitsausweis geschaffen wurde, fehlen in diesem Bereich Kriterien für die Auswahl phytotherapeutischer Leistungserbringer.

Seit dem 1. Juli 2005 sind die komplementärmedizinischen Methoden TCM (Chinesische Phytotherapie), Homöopathie, Neuraltherapie, anthroposophisch-erweiterte Medizin und Phytotherapie aus der Grundversicherung ausgeschlossen. Die Akupunktur ist davon nicht betroffen. Damit sind drei Fähigkeitsausweise vollständig, ein Fähigkeitsausweis teilweise und ein Fachgebiet ohne Fähigkeitsausweis (Phytotherapie) in die Zusatzversicherung relegiert.

\section{Qualitätsstandards}

Eines der wichtigsten Ziele der ärztlichen Berufsverbände ist die Schaffung, Überprüfung und Weiterentwicklung der Fachkompetenz ihrer Mitglieder. Dies gilt ohne Abstriche auch für den Bereich der Zusatzversicherungen; es gibt nur eine Qualität. Die Qualitätskriterien der FMH und der in der UNION organisierten Gesellschaften bilden die Garantie dafür, dass diese von der Ärzteschaft überwacht und weiterentwickelt werden. Nach übereinstimmender Auffassung von FMH und UNION bieten sich die bestehenden Fähigkeitsausweise der Komplementärmedizin zur Anerkennung in der Zusatzversicherung an: Was in der ehemaligen Grundversicherung gesetzliche Vorgabe war, ist als Qualitätsstandard auch in der Zusatzversicherung sinnvoll. Selbstverständlich ist es den Zusatzversicherern unbenommen, auch weitere in- und ausländische Qualifikationen zu berücksichtigen. Hinweis: Anbieter von Qualitätsstandards bei den nichtärztlichen Therapeuten (EMR) sind nicht daran interessiert, im Bereich der ärztlichen Komplementärmedizin tätig zu werden.
Dies bedeutet auch, dass die bestehenden Fähigkeitsausweise innerhalb der FMH in ihrer Gültigkeit unverändert weiterbestehen und gepflegt werden. Die stets aktualisierte Liste aller Träger von Fähigkeitsausweisen befindet sich auf der Website der FMH unter www.fmh-index.ch.

\section{Die Fachdisziplinen im einzelnen}

Fähigkeitsausweis Akupunktur - TCM (ASA) Für die Akupunktur sind alle Inhaber des Ausweises innerhalb der Grundversicherung weiterhin abrechnungsberechtigt; es gelten die Limitationen der betroffenen TARMED-Positionen. Kombinationen mit anderen Positionen der Grundversorgung sind möglich. Für die Überwachung wurde eine neue Position geschaffen (00.1735). Bei einem Patienten, der nach Erreichen der Limitationen eine Weiterführung der Akupunkturbehandlung benötigt, kann diese Zusatzleistung über den Patienten als Selbstzahler bzw. über seine Zusatzversicherung abgerechnet werden.

Bei der traditionellen chinesischen Medizin kommt die Zusatzversicherung bzw. die Patientin als Selbstzahlerin zum Zug. Als Qualitätskriterium steht der gleiche Fähigkeitsausweis zur Verfügung.

\section{Fähigkeitsausweise Homöopathie (SVHA),} Neuraltherapie (SANTH) und anthroposophisch-erweiterte Medizin (VAOAS)

Diese Ausweise bilden als Qualitätsstandard im jeweiligen Fachgebiet ebenfalls ein adäquates Kriterium für die Zusatzversicherer.

\section{Phytotherapie (SMGP)}

Die Schweizerische medizinische Gesellschaft für Phytotherapie hat eigene Qualitätsstandards erarbeitet. Diese werden von der SMGP und der UNION verwaltet und dienen als Qualitätsmerkmal für spezifisch ausgebildete Ärztinnen und Ärzte. Die Standards entsprechen den generellen Vorgaben, die für die Fähigkeitsausweise der FMH gelten. 


\section{Abrechnung im Zusatzversicherungs- bereich}

Es sei an dieser Stelle an die spezifischen Erfordernisse, die zum Teil von den gesetzlichen Bedingungen des KVG abweichen, erinnert:

- Der Leistungserbringer ist in der Wahl seines Honoraransatzes nicht an Tarife gebunden.

- Tarife, Empfehlungen oder Absprachen unter den Leistungserbringern sind gemäss Kartellgesetz nicht erlaubt. Dies führt faktisch dazu, dass die entsprechenden Fachdisziplinen auch keinen Korridor für den Honoraransatz angeben dürfen. Die für solche Honorarempfehlungen oder -absprachen vorgesehenen Strafen sind - nach ärztlichem Verständnis drakonische Geldbussen. Beschwerden in bezug auf überhöhte Rechnungsstellungen, selbst wenn diese nach gesundem Menschenverstand als gerechtfertigt beurteilt werden müssten, können somit von den Fachdisziplinen und auch den kantonalen Ärztegesellschaften nicht weiterverfolgt werden. Zuständig für Honorarstreitigkeiten ausserhalb der Grundversicherung ist der Zivilrichter.
- Ausgleichend hat der Gesetzgeber dafür vorgesehen, dass der Honoraransatz dem Patienten/Klienten vor der Leistungserbringung bekanntgegeben werden muss; konkret bedeutet dies u.a., dass die zur Anwendung gelangenden Ansätze im Wartezimmer angeschlagen sein müssen.

- Die Patientin muss darüber aufgeklärt werden, dass die Behandlung nicht im Rahmen ihrer Grundversicherung übernommen wird und dass die ihr jetzt bekannten Honoraransätze zum Zuge kommen.

- Sind diese Bedingungen erfüllt, so ist es der Patientin überlassen und in ihrer Verantwortung, ob sie das Honorar selbst oder über die Zusatzversicherung begleicht.

\section{Ausblick}

Mit der Beibehaltung der bewährten, von der FMH anerkannten Fähigkeitsausweise und der damit verbundenen Qualitätsstandards bietet die Ärzteschaft den Zusatzversicherern eine unbürokratische, qualitativ hochstehende und verlässliche Qualitätssicherung im Bereich der Komplementärmedizin an. 